\title{
Detection of Significant Prostate Cancer According to Anatomical Areas of Sampling Cores Obtained with Transrectal Systematic 12-Core Biopsy
}

\author{
Hiromoto Tei Hideaki Miyake Ken-ichi Harada Masato Fujisawa \\ Division of Urology, Kobe University Graduate School of Medicine, Kobe, Japan
}

\section{Key Words}

Prostate cancer • Biopsy • Radical prostatectomy •

Significant cancer

\begin{abstract}
Background: To analyze the diagnostic performance of 12core biopsy in detecting significant prostate cancer (PCa). Patients and Methods: This study included 206 PCa patients who underwent transrectal 12-core biopsy followed by radical prostatectomy. Radical prostatectomy specimens were anatomically divided into 12 areas according to the sampling cores, and the existence of significant cancer, defined by a tumor volume $>0.5 \mathrm{ml}$, was investigated. The detection rate of significant cancer in each area was calculated as follows: the number of positive core biopsies/the number of areas containing significant cancer $\times 100$. Results: The overall detection rate of significant cancer in all areas was $53.6 \%$. The detection rate was significantly higher in the standard sextant cores than in the additional 6 cores in patients with prostate-specific antigen $\geq 10 \mathrm{ng} / \mathrm{ml}$, clinical stage $\geq \mathrm{T} 2$, or biopsy Gleason score $\geq 7$, but not in those with prostate-specific antigen $<10 \mathrm{ng} / \mathrm{ml}$, clinical stage T1c, or biopsy Gleason score $\leq 6$. Conclusions: Approximately half of the significant cancers were not accurately detected, and the detection rates in biopsy cores other than the sextant cores appeared to be significantly lower in PCa patients with aggressive features.

Copyright $\odot 2014$ S. Karger AG, Basel
\end{abstract}

\section{KARGER}

Fax +4161306 1234

E-Mail karger@karger.ch

www.karger.com
(C) 2014 S. Karger AG, Basel

$1015-9770 / 14 / 0082-0091 \$ 38.00 / 0$

Accessible online at:

www.karger.com/cur

\section{Introduction}

Prostate cancer $(\mathrm{PCa})$ is the most frequently diagnosed malignancy and second leading cause of cancer-specific deaths in men in Western industrialized countries [1]. The introduction of a serum prostate-specific antigen (PSA) assay combined with a digital rectal examination as a screening tool for PCa has resulted in a marked increase in the number of patients subsequently undergoing transrectal ultrasound (TRUS)-guided needle biopsy, which is regarded as a mainstay in the diagnosis of PCa [2]. The sextant technique, which targets the peripheral zone (PZ), including the apex, middle, and base of the bilateral lobe, has become the standard method used for prostate biopsies and has significantly improved the detection of PCa [3]. However, this procedure has been shown to underestimate the presence of malignant disease, with false-negative rates ranging $\mathrm{m}$ approximately 10 to $30 \%$ [4]. Therefore, alternative prostate biopsy schemes, intended to perform more extensive systematic sampling, have been considered in order to improve PCa detection rates [4-7]. Twelve cores have routinely been taken from each patient undergoing TRUS-guided biopsy at our institution; 8 cores from the PZ, including the standard sextant cores and 2 cores from the anterior lateral horns (ALHs), 2 cores from the transition zones (TZs), and an additional 2 cores from the dorsal apex (DA). Taking 12 cores was shown to result in a comparatively satisfactory outcome in the cancer detection rate [8].

A number of studies have so far assessed the significance of prostate biopsy in the detection of $\mathrm{PCa}$ in each patient [2-8]; however, investigating the diagnostic ac-

Hideaki Miyake

Division of Urology, Kobe University Graduate School of Medicine

7-5-1 Kusunoki-cho, Chuo-ku

JP-6500017, Kobe (Japan)

E-Mail hideakimiyake@ hotmail.com 
Table 1. Patient characteristics

\begin{tabular}{ll}
\hline Item & Value \\
\hline Age $(\text { years })^{*}$ & $67.5 \pm 5.9$ \\
Serum PSA $(\mathrm{ng} / \mathrm{ml}) *$ & $10.3 \pm 7.7$ \\
Clinical stage $(\%)$ & $68(33.0)$ \\
$\quad$ T1c & $138(67.0)$ \\
T2 & $74(35.9)$ \\
Biopsy Gleason score $(\%)$ & $108(52.4)$ \\
$\geq 6$ & $24(11.7)$ \\
7 & $154(74.8)$ \\
$\geq 8$ & $52(25.2)$ \\
Pathological stage $(\%)$ & $61(29.6)$ \\
pT2 & $130(63.1)$ \\
pT3 & $15(7.3)$ \\
Gleason score $(\%)$ & \\
$\geq 6$ & \\
7 & \\
$\geq 8$ & \\
\hline PSA = Prostate-specific antigen. $*$, values are expressed as \\
the mean \pm SD.
\end{tabular}

curacy of all malignant foci present in the prostate has become important gland in several situations associated with the treatment of patients with PCa [9-12]. For example, the recent introduction of robotic techniques has allowed for the precise preservation of the neurovascular bundles, resulting in a significant increase in the proportion of patients undergoing nerve-spring radical prostatectomy (RP), which is commonly indicated in consideration of the anatomical localization of tumor foci in the prostate [10]. The implications for focal therapies targeting the affected side have also been determined by the localized pattern of PCa foci based on the pathological outcomes of positive biopsy cores [11]. Considering these findings, it would be of interest to investigation the detection rate of $\mathrm{PCa}$ according to anatomical areas in the prostate; therefore, we performed a mapping evaluation of RP specimens obtained from patients diagnosed following 12-core systematic biopsies in the present study, and assessed the detection rate of significant $\mathrm{PCa}$ in each area from which the biopsy core was taken.

\section{Patients and methods}

\section{Patients}

This study included a total of 206 patients who were diagnosed with clinically localized PCa by systematic 12-core biopsies and subsequently underwent RP without any neoadjuvant therapies
Table 2. Cancer detection rate according to the anatomical area of sampling cores

\begin{tabular}{lll}
\hline & $\begin{array}{l}\text { No. of positive cores/ } \\
\text { No. of significant cancers }\end{array}$ & $\begin{array}{l}\text { Cancer detection } \\
\text { rate }(\%)\end{array}$ \\
\hline $\begin{array}{l}\text { Standard sextant } \\
\text { cores }\end{array}$ & \\
$\quad$ Apex & $93 / 153$ & \\
Middle & $101 / 176$ & 60.8 \\
Base & $51 / 94$ & 57.4 \\
$\quad$ Total & $245 / 423$ & 54.3 \\
Other biopsy & & 57.9 \\
cores & & \\
ALH & $50 / 114$ & \\
TZ & $52 / 104$ & 43.9 \\
DA & $70 / 137$ & 50.0 \\
Total & $172 / 355$ & 51.1 \\
Overall biopsy & & 48.5 \\
cores & $417 / 778$ & 53.6 \\
& & \\
\hline
\end{tabular}

$\mathrm{ALH}=$ Anterior lateral horn; $\mathrm{TZ}=$ transition zone; $\mathrm{DA}=$ dorsal apex. *, significantly higher than the cancer detection rate in other biopsy cores $(\mathrm{p}=0.0081)$.

at our institution between January 2008 and December 2012. Informed consent to perform this study was obtained from all patients, and the study design was approved by the Research Ethics Committee of our institution.

\section{TRUS-Guided Prostate Biopsy}

The prostate biopsy procedure performed in this series has been described previously [8]. Briefly, after a sacral block, transrectal biopsies of the prostate were taken with a spring-loaded biopsy gun (Biopty, CR Bard, Covington, GA) and 18-guage Tru-cut biopsy needle under TRUS guidance using a model 1846 console with a multiplane transducer (Model 8551; Bruel \& Kajer, Naerum, Denmark). Our 12-core based biopsy strategy was as follows: specimens were obtained from 8 cores from the PZ consisting of the apex, middle, and base of the PZ, and ALH, which represented the extreme lateral and anterior PZ surrounding the $\mathrm{TZ}$, in each lobe, 2 cores from the TZ, and additional 2 cores from the DA.

\section{Assessment of RP Specimens}

$\mathrm{RP}$ and bilateral pelvic lymphadenectomy were performed based on the surgical procedure described by either Walsh [13] or Patel et al. [14]. Resected prostate specimens were prepared using the whole-mount technique, in which the surface was inked and fixed in neutral formalin solution. After fixation, the apex and base were amputated and then serially sectioned along the vertical parasagittal plane. The seminal vesicles were sectioned parallel to their junction with the prostate and entirely submitted for evaluation. The remaining specimen was serially sectioned perpendicularly to the long axis of the gland from the apex to the base at 3-mm intervals. Each section was stained with hematoxylin and 
Table 3. Cancer detection rate according to several clinical parameters

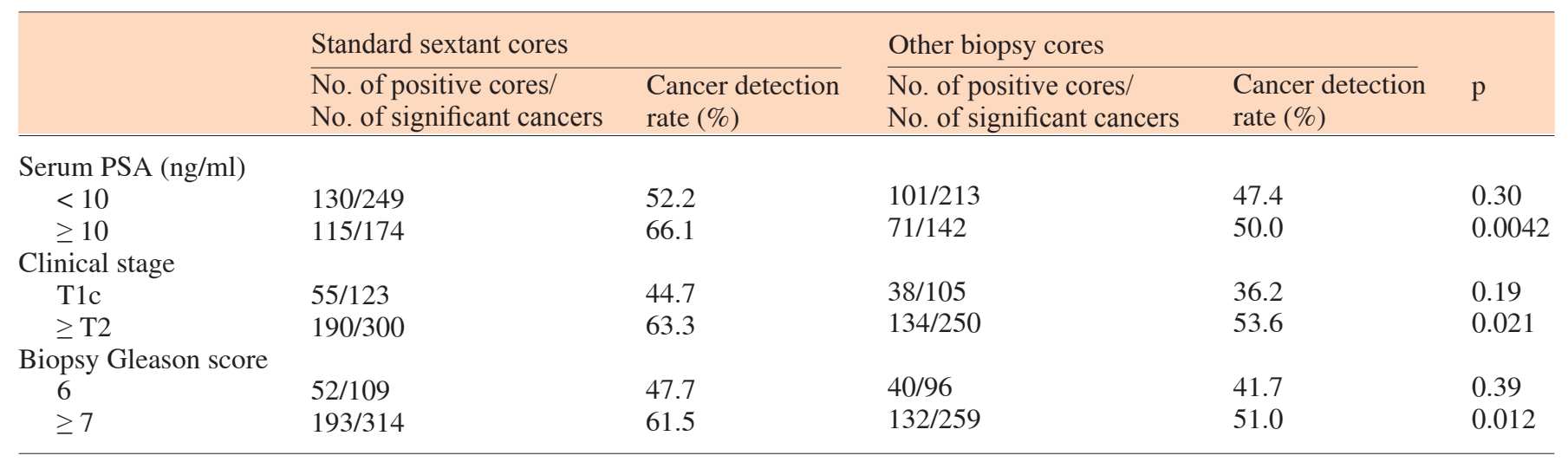

PSA $=$ Prostate-specific antigen.

eosin, and examined for the location of cancer, capsular penetration, and seminal vesicle invasion. All pathological specimens were examined in the present study under the guidance of a single pathologist according to the 2002 TNM classification system.

\section{Calculation of the Cancer Detection Rate}

Each tumor volume detected in the mapping study of RP specimens was determined as described previously [15]. Briefly, the maximum tumor area of the single tumor focus was d using a digital planimeter (Uchida Yoko, Tokyo, Japan), and the tumor volume was calculated as the sum of surface areas for that tumor multiplied by the thickness of the prostate slice. Clinically significant cancer was regarded as a tumor greater than $0.5 \mathrm{ml}$ according to the criterion advocated by Stamey et al. [16]. RP specimens were anatomically divided into 12 areas according to the sampling cores of the prostate biopsy, and the existence of significant cancer, defined by a tumor volume $>0.5 \mathrm{ml}$, was examined. The detection rate of significant cancer in each area was calculated as follows: the number of positive core biopsies/the number of areas containing significant cancer $\times 100$.

\section{Statistical Analysis}

All statistical analyses were performed using Statview 5.0 software (Abacus Concepts, Inc., Berkeley, California), and p values less than 0.05 were considered significant. Differences in the cancer detection rates between 2 categories were analyzed using the chi-square test.

\section{Results}

The clinicopathological characteristics of the 206 patients included in this study are summarized in table 1. As shown in table 2, pathological examinations of the 12 core-biopsy specimens identified a total of 417 cancer-positive cores, while the mapping study of RP specimens detected 778 areas containing significant cancer-

Transrectal Systematic 12-Core Prostate Biopsy for Prostate Cancer ous foci. Therefore, the overall cancer detection rate in all 12 areas was $53.6 \%$. Furthermore, the detection rate of significant cancer was significantly higher in the standard sextant cores than in the additional 6 cores.

We then investigated the relationship between the detection rate of significant cancer and major clinical parameters available prior to RP. As shown in table 3, the cancer detection rate was significantly higher in the standard sextant cores than in the additional 6 cores in patients with PSA $10 \mathrm{ng} / \mathrm{ml}$, clinical stage T2, or biopsy Gleason score 7, but not in those with PSA $<10 \mathrm{ng} / \mathrm{ml}$, clinical stage T1c, or biopsy Gleason score 6 .

\section{Discussion}

The most accurate way to detect cancer foci inside the prostate gland is surgical removal followed by a pathological examination of the entire gland. Since it is impossible to clinically apply this approach to each patient suspected of $\mathrm{PCa}$, prostate biopsy is accepted as the best diagnostic technique to detect PCa [4]. TRUS-guided sextant biopsy, introduced by Hodge et al. [3], was the traditional method used in the diagnosis of patients with PCa. However, this procedure has been replaced over the past 2 decades by strategies that intend more extensively sampling from alternative areas of the prostate because many studies reported improved rates of $\mathrm{PCa}$ detection with an increase in the number of biopsy cores [4-7]. Therefore, TRUS-guided biopsy targeting 10-18 cores has become a common approach for diagnosing $\mathrm{PCa}$ in many high volume centers [4]. Systematic biopsies that 
take 12 cores from each patient are routinely performed at our institution, and have yielded a comparatively favorable outcome with a cancer detection rate of $35.0 \%$ [8].

Most studies that have evaluated the significance of prostate biopsy have focused on the detection of $\mathrm{PCa}$ in each patient [2-8]; however, it is also important to assess whether each cancerous lesion is exactly diagnosed considering the recent trend in therapeutic options for patients with early localized PCa. In facts, information on the anatomical localization of tumor foci in the prostate has had crucial effects on decision-making in several clinical situations [9-12]. In this study, therefore, we precisely mapped $\mathrm{PCa}$ in RP specimens from PCa patients diagnosed by 12-core systematic biopsies, and analyzed the detection rate of significant $\mathrm{PCa}$ according to 12 anatomical areas divided by the distribution of biopsy cores in the prostate.

We identified 778 areas containing significant PCa by mapping RP specimens; however, only 417 biopsy cores were diagnosed with $\mathrm{PCa}$ by pathological examinations. This outcome showed that the overall detection rate of significant PCa in all 12 anatomical areas was 53.6\%. Few studies have evaluated the ability of prostate biopsies to detect $\mathrm{PCa}$ after the presence of malignant lesions has been pathologically confirmed in the prostate $[17$, 18]. For example, Fink et al. [17] performed ex-vivo sextant biopsy on removed prostate specimens with biopsy-proven cancer, and showed that approximately $10 \%$ of all cancers, most of which were clinically significant, were not diagnosed, even though 20-core biopsy cores were taken. However, these studies did not address the diagnostic accuracy of each cancerous lesion; hence, to the best of our knowledge, this study is the first to report cancer detection rates calculated based on the finding of an individual significant $\mathrm{PCa}$.

It is of interest to compare cancer detection rates according to the 12 anatomical areas divided by the sampling manner of prostate biopsy. In this study, the cancer detection rate was shown to be significantly higher in the sextant biopsy cores than in the additional 6 cores. In fact, the cancer detection rates in the apex, middle, and base of the $\mathrm{PZ}$ were all $>50 \%$, whereas those in the $\mathrm{ALH}, \mathrm{TZ}$, and DA were all $\leq 50 \%$. The lower detection rates of significant $\mathrm{PCa}$ in areas other than the sextant cores can be explained as follows: it is technically difficult to obtain optimal biopsy specimens from the ALH and DA through a transrectal approach in some patients $[8,19]$, while, the number of cores taken from the TZ may be insufficient, particularly in patients with larger prostates, considering a sample amount with 2 biopsy cores of approximately $30 \mathrm{~mm}^{3}$ [18]. These findings suggest that it might be a promising approach to combine the transperineal approach with the transperineal approach and /or increase the number of biopsy cores targeting the $\mathrm{TZ}$ in order to improve the detection rate of significant PCa.

Another point of interest is the relationship between the cancer detection rate according to the anatomical areas and the major clinical parameters. The cancer detection rate was significantly higher in the standard sextant cores than in the additional 6 cores in patients with higher serum PSA, more advanced clinical stage, or greater biopsy Gleason score. Although it is difficult to explain the reason for this trend, these findings suggest that tumor foci with features characteristic to aggressive phenotypes are likely to be misdiagnosed in cores other than those obtained using the sextant biopsy procedure; therefore, it is of importance to further evaluate the presence of tumor foci using alternative diagnostic tools, such as magnetic resonance imaging, in patients with $\mathrm{PCa}$ characterized by aggressive features.

There were several limitations to this study. Firstly, this was a retrospective study, and the sample size of 206 men in such a common disease as $\mathrm{PCa}$ was not large enough to draw definitive conclusions on the issues assessed in this study. Secondly, preoperative TRUS-guided 12-core prostate biopsies were not performed by a single physician, which may have influenced the current results. Thirdly, this study included only men with positive prostate biopsies and excluded men with false-negative initial biopsies; hence, the actual cancer detection rate may be much lower, and further studies are required to resolve this problem. Fourthly, despite the comparable concordance rates between biopsy and RP Gleason scores to those in previous studies [20,21], the impact of inaccurate Gleason grading, particularly that of downgrading of the biopsy Gleason score 8 , on the present findings should be considered. Finally, although an investigation on the significance of additional parameters, such as patient age and prostate volume, was conducted, there was no significant association between the cancer detection rate and these factors (data not shown). However, it remains important to further examine the impacts of factors that may be potentially associated with the cancer detection rate in order to more clearly understand the diagnosis of each tumor foci using systematic biopsies.

In conclusion, the results of the present study suggest that approximately half of the significant cancers were not accurately detected by systematic TRUS-guided 12- 
core biopsies, and the detection rates of significant $\mathrm{PCa}$ in cores other than those of traditional sextant biopsy were significantly lower in patients with PCa having aggressive features. Therefore, a novel strategy needs to be developed to sample biopsy cores from areas different to those obtained with the sextant biopsy procedure in order to improve the detection rate of significant $\mathrm{PCa}$.

\section{References}

1 Jemal A, Siegel R, Xu J, Ward E: Cancer statistics, 2010. CA Cancer J Clin 2010;60: 277-300.

-2 Serag H, Banerjee S, Saeb-Parsy K, Irving S, Wright K, Stearn S, Doble A, Gnanapragasam VJ: Risk profiles of prostate cancers identified from UK primary care using national referral guidelines. Br J Cancer 2012; 106:436-439.

- 3 Hodge KK, McNeal JE, Terris MK, Stamey TA: Random systematic versus directed ultrasound guided transrectal core biopsies of the prostate. J Urol 1989;142:71-74.

-4 Eichler K, Hempel S, Wilby J, Myers L, Bachmann LM, Kleijnen J: Diagnostic value of systematic biopsy methods in the investigation of prostate cancer: a systematic review. J Urol 2006;175:1605-1612.

-5 Babaian RJ, Toi A, Kamoi K, Troncoso P, Sweet J, Evans R, Johnston D, Chen M: A comparative analysis of sextant and an extended 11-core multisite directed biopsy strategy. J Urol 2000;163:152-157.

6 Naughton CK, Miller DC, Mager DE, Ornstein DK, Catalona WJ: A prospective randomized trial comparing 6 versus 12 prostate biopsy cores: impact on cancer detection. J Urol 2000;164:388-392.

7 Jiang X, Zhu S, Feng G, Zhang Z, Li C, Li H, Wang $\mathrm{C}$, $\mathrm{Xu}$ Y: Is an initial saturation prostate biopsy scheme better than an extended scheme for detection of prostate cancer? A systematic review and meta-analysis. Eur Urol 2013;63:1031-1039.

8 Miyake H, Harada K, Inoue TA, Takenaka A, Hara I, Fujisawa M: Additional sampling of dorsal apex on systematic prostate biopsy: impact on early detection of prostate cancer. Urology 2007;69:738-742.
9 Bolenz C, Gierth M, Grobholz R, Kopke T, Semjonow A, Weiss C, Alken P, Michel MS, Trojan L: Clinical staging error in prostate cancer: localization and relevance of undetected tumour areas. BJU Int 2009;103:11841189.

10 Srivastava A, Chopra S, Pham A, Sooriakumaran P, Durand M, Chughtai B, Gruschow S, Peyser A, Harneja N, Leung R, Lee R, Herman M, Robinson B, Shevchuk M, Tewari A: Effect of a risk-stratified grade of nerve-sparing technique on early return of continence after robot-assisted laparoscopic radical prostatectomy. Eur Urol 2013;63:438-444.

11 Sinnott M, Falzarano SM, Hernandez AV, Jones JS, Klein EA, Zhou M, Magi-Galluzzi C: Discrepancy in prostate cancer localization between biopsy and prostatectomy specimens in patients with unilateral positive biopsy: implications for focal therapy. Prostate 2012;72:1179-1186.

12 Falzarano SM, Zhou M, Hernandez AV, Moussa AS, Jones JS, Magi-Galluzzi C: Can saturation biopsy predict prostate cancer localization in radical prostatectomy specimens: a correlative study and implications for focal therapy. Urology 2010;76:682-687.

13 Walsh PC: Radical prostatectomy: a procedure in evolution. Semin Oncol 1994;21:662671.

14 Patel VR, Thaly R, Shah K: Robotic radical prostatectomy: outcomes of 500 cases. BJU Int 2007;99:1109-1112.

15 Muramaki M, Miyake H, Kurahashi T, Takenaka A, Fujisawa M: Characterization of the anatomical extension pattern of localized prostate cancer arising in the peripheral zone. BJU Int 2010;105:1514-1518.
16 Stamey TA, Freiha FS, McNeal JE, Redwine EA, Whittemore AS, Schmid HP: Localized prostate cancer. Relationship of tumor volume to clinical significance for treatment of prostate cancer. Cancer 1993;71(3 suppl):933-938.

17 Fink KG, Hutarew G, Lumper W, Jungwirth A, Dietze O, Schmeller NT: Prostate cancer detection with two sets of ten-core compared with two sets of sextant biopsies. Urology 2001;58:735-739.

18 Serefoglu EC, Altinova S, Ugras NS, Akincioglu E, Asil E, Balbay MD: How reliable is 12-core prostate biopsy procedure in the detection of prostate cancer? Can Urol Assoc J 2012;2:1-6.

19 Miyake H, Sakai I, Ishimura T, Hara I, Eto $\mathrm{H}$ : Significance of cancer detection in the anterior lateral horn on systematic prostate biopsy: the effect on pathological findings of radical prostatectomy specimens. BJU Int 2004;93:57-59.

20 Epstein JI, Feng Z, Trock BJ, Pierorazio PM: Upgrading and downgrading of prostate cancer from biopsy to radical prostatectomy: incidence and predictive factors using the modified Gleason grading system and factoring in tertiary grades. Eur Urol 2012;61:1019-1024.

- 21 Kvale R, Moller B, Wahlqvist R, Fossa SD, Berner A, Busch C, Kyrdalen AE, Svindland A, Viset T, Halvorsen OJ: Concordance between Gleason scores of needle biopsies and radical prostatectomy specimens: a population-based study. BJU Int 2009;103:16471654 . 